\title{
AUTOLOGOUS PLATELET - RICH PLASMA (PRP) THERAPY AND CHANGEMENTS OF TOPICAL BIOLOGICAL MARKERS (EGF, VEGF AND MMP12) OF CHRONIC WOUNDS
}

\author{
Nguyen Ngoc Tuan*, Nguyen Tran Ngoc Tu**, Nguyen Tien Dung*
}

\section{ABSTRACT}

Objective: Our aim was to evaluate topical EGF, VEGF, MMP12 concentrations of chronic wounds which after Autologous Platelet - Rich Plasma(PRP) therapy.

Methods: The study conducted a descriptive longitudinal study at the Wound Healing Center of the Vietnam National Burn Hospital from $11 / 2018$ to $2 / 2020$. 24 patients with chronic wounds and aged 18 years old or older were enrolled in this study. These patients were injected autologous PRP at wound bed. We biopsied chronic wound bed tissue for evaluating EGF, VEGF and MMP12 by the Elisa technique at the first, second and third week of follow-up.

Results: After PRP therapy, topical EGF and VEGF concentration increased after 1 week of treatment. MMP12 concentraiton significantly reduced compaired to before PRP therapy.

Conclusion: In addition to PRP therapy had a beneficial effect on cutaneous regeneration and wound healing of the chronic wound. The autologous PRP promoted the wound healing process byincreating topical EGF and VEGF, reducing MMP12 which known as a proinflammatiory mediator.

Keywords: Platelet - Rich Plasma, EGF, VEGF, MMP12, Chronic wound

\section{INTRODUCTION}

Chronic wounds are serious health problems that are drawing attention in

\footnotetext{
* Le Huu Trac National Burn Hospital

** National Institute of Nutrition
}

Responsible person: Nguyen Ngoc Tuan

Email: ngoctuan64@gmail.com

Date of receipt: 29/5/2021

Date of scientific judgment: 01/7/2021

Reviewed date: 05/8/2021 society. In the USA, chronic wounds affect around $3-6$ million people in which with people aged over-65-year-olds account for $85 \%$. The cost for treatment of chronic wounds is estimated at approximately 3 billion USD annually [1], [2]. By 2014, the cost of care for pressure ulcers alone is around 11 billion (USD) annually and the average cost for each ulcer is $500-70.000$ (USD). In the U.K., the annual cost for medical care of wounds is determined at 2.165 billion USD in 2014 (Dowsett C., 2014 [3]). In developed countries, around 1- $2 \%$ of the population would have a permanent chronic injury in their whole life. The cost for chronic wound treatment takes up to 2 $3 \%$ of the total health budget [4]. The consequences as well as complications of chronic injuries are severe, affecting patients' quality of life or even threatening their life, such as: amputation, sepsis, cachexia etc. Chronic wounds have common characteristics, which are disorders in the recreation-healing process, along with systematic background pathological histories. Treatment of chronic ulcers is a sophisticated, lengthy, costly process that requires the application of many methods. Support for cellular and other components' proliferation and epithelization at wound location is one such method. There is a trend to use Platelet rich plasma - PRP in treating chronic ulcers.

PRP has a platelet rate significantly higher than that of plasma in regular blood. When 
the platelet is activated, it leads to the $\alpha$ particles lysis inside the platelet, from there release a number of proteins, including antiinflammatory cytokines, chemokines and dozens of growth factors (GF) which plays an important role in wound healing $[5,6]$. Nowadays, PRP is likely to gain popularity and has been applied into many fields, such as orthopedic, sports, the dental surgery, otorhinorarynology, neurosurgery, ophthalmology, urology, would regeneration, plastic surgery, and cosmetics etc. due to its efficiency, safety, cost-effective preparation with minimal side effects [7]. There is a need for objective indicator research to elucidate the mechanism of PRP at chronic wounds. Therefore, we have conducted this research with the aim to: 1/ Evaluate the change of EGF, VEGF in PRP before and after activation. 2/ Evaluate the change of EGF, VEGF, hydroxyproline, MMP12 concentration in PRP-treated chronic injuries. The results shall be objective proof for the effectiveness of PRP towards treatment of chronic wounds.

\section{SUBJECTS AND METHODS}

\section{Subjects}

The study included 24 patients with chronic injuries, the age of 18 and older. They hospitalited at the Wound Healing Center - Le HuuTrac National Burn Hospital from 11/2018 - 2/2020.

\section{Study inclusion and exclusion criteria}

The patients aged 18 years or older. They were asked to sign an informed consent form to participate in the study. We also excluded patients with HIV positive, hepatitis B, hepatitis C virus, Platelet dysfunction syndrome; severe platelet reduction $\quad<50$ G/l), fibrinogen decrease; severe whole-body condition, requiring positive healing, hemodynamic disorder; sepsis; Infections occur at location of procedure; patients in chemotherapy, with appearance of ulcers due to cancer; pregnancy, lactation; patients do not consent to participate. Relative contraindication: fever; $\mathrm{Hb}<100 \mathrm{~g} / \mathrm{L}$; platelet $<150 \mathrm{G} / \mathrm{L}$ from the study.

Ethical criteria: This study was approved by the the Institutional Ethics Commitee. Patients volunteer to participate on experiment. They could refuse participating at any time.

\section{Study material:}

- PRP extraction Kit: "New-PRP Pro Kit" for extraction of PRP which includes: Specialized blood collection needles and blood collection tubes; Tubes with aseptic anticoagulants; centrifugal tube containing substances to activate platelets. EBA-20 (German) centrifuge. Grade 2 bio-safety cabinet.

- EGF and VEGF testing kit: Protease Inhibitor Cocktail, 50X (G652A, Promega). EGF testing kit: Human EGF ELISA Kit (EK193-96, Multi Sciences); which includes: pre-coated 96-well plate with ready-covered anti-EGF antibodies; standard EGF: recombinant protein in a certain concentration; EGF detectors: EGF specific antibodies attached to biotin; Streptavidin HRP: 100x solution. Assay Buffer (10x): PBS with $0.5 \%$ Tween-20 and 5\% BSA; tetramethyl-benzidine (TMB); sulfuric acid $0.18 \mathrm{M}$ solution; buffer solution for washing (20x): PBS with 1\% Tween-20; Plate Covers (cover for 96-well plate)

VEGF testing kit: Human VEGF ELISA Kit (EK183-96, Multi Sciences) pre-coated 96-well plate anti-VEGF antibodies; Standard VEGF. VEGF detectors: VEGF specific antibodies attached to biotin. 
- MMP-12 testing Kit: Proceed quantitative steps according to instructions by manufacturers on ELISA machine by Diagnostic Automation, United States. Components of testing kit (ab213811 Human MMP-12 ELISA Kit): Micro-elisa antibody plate to MMP-12 people (1 plate of96-well); human regeneration Protein MMP-12 (2 vials), MMP-12 antibodies attached to Biotin $(130 \mu \mathrm{L})$; Avidin-BiotinPeroxidase complex (ABC, $130 \mu \mathrm{L}$ ); solution for sample dilution $(30 \mathrm{~mL})$; Antibody dilution solution $(12 \mathrm{~mL}), \mathrm{ABC}$ dilution solution $(12 \mathrm{~mL})$; TMB $(10 \mathrm{~mL})$; Antireaction solution $(10 \mathrm{~mL})$, Cover (4). Storage temperature: $-20^{\circ} \mathrm{C}$

\section{Procedure}

- Patient selection. Daily dressing change according to procedure until the chronic wound is stable.

- Extraction and injection of PRP in peri wound: Proceed PRP treatment only when there is no caseationor acute infections $[7,8$, $11,12]$. Second injection after 1 week [10].

- EGF and VEGF in PRP testing before and after platelet activation at both PRP reception times.

- Topical EGF, VEGF and MMP12 of chronic wound were valuated before PRP injection, after 1 week and 2 weeks.

PRP harvest (according to the guidline of GeneWorld $^{\odot}$ ).

- Phase 1 (deactivated PRP collection): We harvested $28 \mathrm{ml}$ of peripheral blood. Centrifuge the blood tube for the first time at $2.000 \mathrm{rpm} \times 10$ minutes to separate the plasma, red blood cells, and platelets. Gently suck the yellow plasma into the centrifugal tube labeled PLASMA (total volume of about $12-14 \mathrm{~mL}$ ). Centrifuge the PLASMA tube for the second time at $3500 \mathrm{rpm} \times 5$ minutes, the fluid harvested is split into 2 layers. Gently suck the upper part away, leaving $6 \mathrm{ml}$ PRP at the bottom. This is deactivated PRP.

- Phase 2: PRP was activated by $\mathrm{CaCl}_{2}$ : Continue to suck $6 \mathrm{ml}$ PRP into the tube which contains $\mathrm{CaCl}_{2}$, simultaneously adding and stirring for 2-5 minutes until a condensed block appears. Use pipet to spin until the block is separated from the tube walls. Wait until the block shrinks down completely (515 minutes), then remove the block. The final product contains $4-5 \mathrm{ml}$ of activated PRP solution, which has a clear yellow color. Suck the entire solution into a sterilized cylinder for wound injection.

\section{Inject PRP into chronic wound site}

PRP was injected around the wound into the peri-wound and wound bed tissue [10]. The injection of PRP is executed in 10-30 minutes after PRP collection. After 5-7 days since first injection, the second PRP injection will be performed. This study was shown, platelets in PRP after activation released about $70 \%$ of GFs in the first 10 minutes; the release of GFs is completed after $1 \mathrm{~h}$. The platelets continue to synthesize the GFs from reserves for at least another 7 days $[6,7,11]$

\subsubsection{Clinical signs monitoring} length.

Age; gender; comorbidities; hospital

- At chronic wound site: we were mornitored and recorded the changement of wound bed, wound fluid (color and amount); edge characteristics

\subsubsection{Mesuring Methods of wound- healing markers \\ 2.3.5.1. EGF and VEGF}

- Technique principles: Following the Elisa reaction method. Quantify the presence of an antibody/antigen by the "sandwich" method. A layer of specific antibodies for EGF/VEGF is covered on well plate. Base 
samples, test samples and antibodies are put into wells if EGF/VEGF receptor biotinsare detected. EGF/VEGF will attach itself to the antibodies at well bottom and with biotin binding detection antibodies after incubation. Remove all specific non-adhesive, add streptavidin - HRP. Wash and add substrate solution into the wells. Color-creating reactions occur, of which the intensity of the color matches the EGF/VEGF concentration. Stop the reaction with another solution. Measure the color intensity, then calculate the EGF/VEGF concentration in the sample.

\section{- Mesurement of EGF in PRP}

+ Preparing samples and testing solutions: PRP samples before activation: after phase 1 of PRP collection, 6ml PRP is collected: Suck about 50 $\mu 1$. PRP samples after activation: at the end of PRP creation process, the final product contains $4-5 \mathrm{ml}$ of a clear yellow solution. Suck about 50 $\mu 1$. The samples are stored at $-80^{\circ} \mathrm{C}$.Before testing, the samples are defrosted in iced water, at a temperature of $18-25^{\circ} \mathrm{C}$.

+ Dilute for testing; create solution; mix antibodies and HRP-washed solution following procedure.

+ Proceed with ELISA reaction for quantifying EGF according to instructions. Measure optical density (OD) at a wavelength of $450 \mathrm{~nm}$ (in 30 minutes after Stopping Solution). Regularize at a wavelength of 570 or $630 \mathrm{~nm}$. Calculate the results: based on the relation between the quantities of standard and EGF samples, develop standard line, identify the EGF concentration $(\mathrm{pg} / \mathrm{ml})$ using a specialized computer software.

- Mesurement of VEGF in PRP: Similar technique to quantifying EGF, replacing reference standard material with VEGF and VEGF antibodies.
- Mesurement of EGF and VEGF in wound tissue: Acquire skin tissue specimens with a weight of approximately $8-30 \mathrm{mg}$ with a Shimadzu scale, AUW220D, Japan. Add Protease Inbibitor Cocktail 1X at a ratio of $1 \mathrm{mg}$ (skin tissue) 10 $\mu 1$. Crush the tissue with a Homogenizer with a speed of $2800 \mathrm{~g} x$ 01 minute, place samples in ice. Centrifuge, collect the samples with MIKRO 200RHettech (Germany) after crushing at $10.000 \mathrm{~g}$ for 10 minutes at $4^{\circ} \mathrm{C}$, collect the fluid, split into 2 tubes, storage $-80^{\circ} \mathrm{C}$. Use the fluid to run the ELISA reaction; steps of procedure are the same as quantifying EGF/VEGF in PRP stated above.

\subsubsection{Mesurement of hydroxyprolin in} chronic wound tissue

We applied Stegemann's method for mesuring hydroxyprolin

- Principle: Use acid $\mathrm{HCl}$ 6N/temperature to full yhydrolyze collagen in skin samples to create amino acid. Oxidizehydroxyproline with chloramine $\mathrm{T}(\sim 7 \%)$ in citrate buffer to create pyrol derivative. Have pyrol derivative react with paradimethylamine benzaldehyde to create color complex, maximum absorption at a wavelength of $\sim 560 \mathrm{~nm}$. The absorption rate of color complex is in linear rate with thehydroxyproline concentration in the sample.

- Preparing sample: Weigh the skin sample (approximately 2mg), put into test tubes. Add $2 \mathrm{ml}$ of $\mathrm{HCl} 6 \mathrm{~N}$ solution, seal the pot then incubate at $115^{\circ} \mathrm{C} / 24 \mathrm{~h}$. After 24 hours, proceed to hydrolyze (skin sample is completely dissolved), then vaporize the acid by steaming. Use $10 \mathrm{ml}$ of pure water to clean the hydrolysis tube and continue vaporizing (redo the process again). Finally, add $2 \mathrm{ml}$ of pure water, move samples to a clean test tube for measuring. 
- Measuring process: Use standard hydroxyproline $10 \mathrm{mg} / \mathrm{L}$ diluted into standard range with a concentration rate of 2,0 - 4,0 $6,0-8,0 \mathrm{mg} / \mathrm{L}$. Put $2 \mathrm{ml}$ of standard sample into the test tube, add $1 \mathrm{ml}$ of testing substance A, shake and leave at $20^{\circ} \mathrm{C}$; after 20 minutes add $2 \mathrm{ml} \mathrm{HClO}_{4} 4 \mathrm{M}$, shake and leave for 5 minutes then add $1 \mathrm{ml}$ of testing substance B. Steam at $60^{\circ} \mathrm{C}$ in 15 minutes, take out and leave to cool down to room temperature. Measure absorption at a wavelength of $560 \mathrm{~nm}$ (solution is pinkpurple) compared to white (includes A, B testing substances and $\left.\mathrm{HClO}_{4}\right)$. After a baseline has been made, go through the same reactions with the analyzing samples, difference in $1 \mathrm{ml}$ of standard solution (prepared above), add $1 \mathrm{ml}$ of pure water.

- Calculate results: hydroxyproline concentration $=(\mathrm{C} \times \mathrm{F}) / \mathrm{m}(\mathrm{mg} / \mathrm{g})$. In which: $\mathrm{C}$ is the concentration measured based on the baseline; F: dilution coefficient (If done as above, $\mathrm{F}=4)$; $\mathrm{m}$ : original sample weight (mg).

\subsubsection{Mesurement of MMP12}

- Method of collecting samples: Biopsy sample is extracted at wound edge, leave inside a $1,5 \mathrm{ml}$ tube containing $1 \mathrm{ml}$ of trizol solution, store at $-20{ }^{0} \mathrm{C}$, then decrease to -80 ${ }^{0} \mathrm{C}$ until experiment. Each tissue sample has an average size: $3-4$ × 3 x 2-3mm.

- Prepare sample; prepare equipment for MMP12-quantifying ELISA experiment following instructions. Note: Preparing MMP12 standard concentration and biotinattached anti MMP12 antibody solution does not exceed 2h; Preparing of ABC complex solution does not exceed 1 hour; $\mathrm{ABC}$ solution and TMB color-creating solution must be kept at $37^{\circ} \mathrm{C}$ x 30 minutes before using.

- Execute Elisa reaction. Measuring optical density (OD) at a wavelength of $450 \mathrm{~nm}$ in 30 minutes since adding anti- reaction solution. Based on the concentration rate of standard samples, create an equation for linear regression between optical density OD (Y) andMMP12 concentration rate (X, $\mathrm{pg} / \mathrm{mL})$.

Linear regression equation is as follows: $\log (\mathrm{Y})=\mathrm{A}^{*} \log (\mathrm{X})^{2}+\mathrm{B} * \log (\mathrm{X})+\mathrm{C}$

\subsubsection{Statistical Analysis}

The results before and after experiment were recorded and compared by using SPSS 20.0 software. The value of $p \leq 0.05$ was considered statistically significant.

\section{III.RESULTS}

\subsection{Study patient}

Mean age: $56,3 \pm 20,7$ (Range:18-85). Male: 14 (58,3\%), female 10 (41,7\%). Comorbidties: TraumaticBrain injury, spinalcordinjuries, extremities fracture: 13 patients $(54,2 \%)$, inability to move the arms or legsin 9 patients (37.5\%); Cardiac diseases (Myocardial infarction, high blood pressure) and stroke: 9 (37.5\%); Diabetes: 7 (29,2\%); high blood pressure: 5 (20,8\%); Intubation: 5 (20,8\%); coma: 4 (16.6\%), and others as meningitis, brain atrophy, Parkinson, gout, systemic lupus erythematosus, peripheral artery disease:12 (49,9\%).

Average diseases /patient: 2,04 \pm 1,07 (1 - 5).

Location of wounds: Thump: 17 patients (70,8\%); greater trochanter, lower hip bones: 11 patients $(45,9 \%)$; toes, and heels: 6 patients (25\%); Other locations: 37,5\%. Pressure ulcers: 20 patients $(83,3 \%)$. Average ulcer number: $2,1 \pm 1,3$ (50\% patients have from 2-5 ulcers); Time of existence: $67,8 \pm$ 71,8 (30 - 360) days; Averagearea of researchulcer: $39,23 \pm 9,18 \mathrm{~cm}^{2}$.

Method of treatment post-PRP injection: Continued dressing change until closed wound: 2 patients $(8,33 \%)$, surgery (skin flaps, skin graft): 22 (91,67\%). Hospital length: $52,7 \pm 26,3$ days. 
Table 3.1. Clinical changement of chronic wound before and after PRP therapy.

\begin{tabular}{|l|c|c|c|}
\hline \multicolumn{1}{c|}{ Variable } & \multicolumn{3}{c|}{ Patients at period } \\
\cline { 2 - 4 } \multicolumn{1}{c|}{ Medium } & T0 & T1 & T2 \\
\hline \multicolumn{1}{|c|}{ Low } & 13 & 0 & 0 \\
\hline Wound amount: High edge:infiltration, callus & 8 & 15 & 5 \\
\hline Epithelization & 3 & 9 & 19 \\
\hline Inflammation, congestion & 17 & 7 & 5 \\
\hline Wound bed: Biofilm & 10 & 18 & 21 \\
\hline Muscle and tendon exposes & 7 & 4 & 0 \\
\hline Granulation tissue: & 15 & 7 & 5 \\
$\quad$ without & 15 & 5 & 3 \\
$\quad$ healthy granulation tissue & & & \\
unhealthy granulationtissue & 10 & 2 & 0 \\
\hline
\end{tabular}

Note: T0: before PRP injection, T1, T2: 1,2 weeks after injection respectively

\subsection{PRP characteristics}

Table 3.2. PRP quality criteria

\begin{tabular}{|l|c|}
\hline \multicolumn{1}{|c|}{ Variable } & Characteristics \\
\hline $\mathrm{pH}$ & $7.38 \pm 0.26$ \\
\hline Number of platelets in PRP before activation & $482,1 \pm 264,5 \mathrm{G} / \mathrm{L}$ \\
Number of platelets in serum & $195,8 \pm 60,1 \mathrm{G} / \mathrm{L}$ \\
\hline Colour & Clear yellow \\
\hline Amount & $4.9 \pm 0.105 \mathrm{~mL}$ \\
\hline Bacterial culture & 0 \\
\hline
\end{tabular}

Table 3.3. EGF and VEGF concentration in PRP

\begin{tabular}{|l|c|c|c|}
\hline \multicolumn{1}{|c|}{ Variable } & Before PRP activation & After PRP activation & P \\
\hline EGF $(\mathrm{pg} / \mathrm{mL})$ & $142,78 \pm 91.56$ & $323,72 \pm 153.73$ & $<0.05$ \\
\hline $\operatorname{VEGF}(\mathrm{pg} / \mathrm{mL})$ & $131.93 \pm 137.73$ & $238.37 \pm 221.53$ & $<0.05$ \\
\hline
\end{tabular}

Table 3.4. EGF and VEGF concentration in wound tissue

\begin{tabular}{|c|c|c|c|}
\hline Variables & Before $1^{\text {st }}$ PRP injection & Before $2^{\text {nd }} P R P$ injection & $\mathbf{P}$ \\
\hline $\mathrm{EGF}(\mathrm{pg} / \mathrm{ml})$ & $0.83 \pm 0.43$ & $1.81 \pm 0.58$ & $<0.05$ \\
\hline $\operatorname{VEGF}(\mathrm{pg} / \mathrm{ml})$ & $4.53 \pm 2.76$ & $12.18 \pm 6.84$ & $<0.05$ \\
\hline
\end{tabular}

Table 3.5. MMP12 and hydroxyproline concentration in wound tissue

\begin{tabular}{|c|c|c|}
\hline Time & Hydroxyproline $(\mathbf{p g} / \mathbf{m l})$ & MMP12(pg/ml) \\
\hline T0 & $4.03 \pm 1.20$ & $4231.04 \pm 2062.833$ \\
\hline T1 & $5.80 \pm 4.04$ & $3792.967 \pm 2185.166$ \\
\hline T2 & $13,01 \pm 6.81$ & $3108.122 \pm 2151.01$ \\
\hline $\mathrm{P}$ & $\mathrm{P}_{0-1}>0,05 ; \mathrm{p}_{0-1} ; \mathrm{p}_{0-2}<0,01$ & $\mathrm{P}_{0-1}<0.05 ; \mathrm{p}_{1-2}<0.05 ; \mathrm{p}_{0-2}<0,001$ \\
\hline
\end{tabular}


IV. DISCUSSION

\subsection{Clinical effects of PRP \\ 4.1.1. Clinical characteristics of study patients}

- Comorbidities: $100 \%$ patients had comorbidities (average of 2,04 $\pm 1,07$ ). Conditions that cause loss or decrease in capability, and control in parts of the body (especially for paralysis of lower body) are brain and spinal injuries. In the United States, $3-5 \%$ of total patients are hospitalized because of the ulcer in spinal cord injuries [13].

- Ulcers had a long existence time and lasting; the number of patients with 2 and above ulcers taking up to $50 \%$ reflects the failure of previous treating methods. Treating chronic wounds with common methods such as care, removal of necrosis, VAC, HBO, etc. can only heal about $50-60 \%[6,14]$.

- Common sites of ulcers: sacrum, joints, lower hip bones. These locations are especially vulnerable to contamination (with urine, etc.), easily dampened, causing difficulties for treating and preventing ulcers.

\subsubsection{Clinical effectiveness of PRP therapy}

- Stimulate wound healing: Chronic wounds all have common features, which are disorders in epithelization and extracellularmatrix (ECM) recreation. Degradation of blood vessels loop becomes old and few, epithelial cells decrease reproduction. Epithelial cells decrease or cease to reproduce, unable to grow. ECM is compromised. Fibroblast is poor or fails to fully function $[1,15]$.

Our research results have shown that wounds before application of PRP treatment method are in a state of disorder during healing. Symptoms of epithelization disorders are shown: edges of wound show no or partial epithelization, border between wound and normal skin clear. Regeneration disorders manifest that the wound background does not havehealthy granulation tissue or unhealthy granulation tissue, many biofilm on the wound bed, muscle and tendon expose. After implementing therapy, the epithelization from edge shown clear, changing the hard infiltration edge, contributing to significantly decreasing wound area $(p<0,05)$. After 2 weeks, number of good granular tissues increased dramatically, $100 \%$ of wounds have granular tissues. Granulation tissues soon covered the tendons, and the muscles. The clinical development is the same as the development of EGF, VEGF, hydroxyproline markers' test development (will be explained in the next part).

- Anti-inflammatory effects: Chronic wounds are injuries that do not heal with its biophysical and age development. Lack or extension of inflamed process slows down healing. Long inflammation creates inflamed cells, slowing GF reactions at inflammation location, increasing MMPs but decreasing protease suppression chemicals; causing rapid GF decrease in chronic wounds. [15, $16,17]$. PRP helps to control inflammations.

On a clinical scale, chronic wounds show long-term inflammation symptoms such as fibrosis, sclerosis [13], biofilm on wound bed, large amount of fluid appear. After implementing PRP treatment for 1-2 weeks, the inflammation is controlled. The amount of fluid, number of wounds with much fluid decreases, infected wounds are also treated $(p<0.05)$. The clinical development is in accordance to the concentration of MMP12 marker (will be explained in the next part). 


\subsection{Changement of concentration of some biomarkers in chonic wound}

Wound healing is a complexed process, which many types of cell participates in, happens on the skin in order to restore the body's line of defense. This process includes the cooperation of some cells such as keratinocytes, fibroblasts, endothelial cells, macrophages and platelets. The movement, infiltration into wound location, reproduction and differentiation of these cells would reach their peak during countering inflammations, creating new tissues and finally sealing wounds. This complicated process is executed and moderated by an intricate network of signals, involving many GFs, cytokines and chemokines [18]. The success in wound healing depends on GFs, which are endogenous signaling molecules that monitor cellular reactions; created by platelets, leukocytes, fibroblasts and epithelial cells $[16,19]$. Even in low concentration rate, GFs still have a clear impact on microenvironmental wounds, leading to the rapid increase in cellular movement, reproduction and differentiation [20]. In which, the most important markers towards wound healing are EGF, VEGF, MMP12, hydroxyproline. Changes of these markers accurately reflects the wound healing process.

\subsubsection{Change in PRP's EGF and VEGF before and after activation}

We harvested PRP by Geneworld $\mathrm{Kit}^{\circledR}$. The number of platelets inside PRP before activation is approximately 2.5 times higher than that in the patients' plasma. Researches have shown, the implementation of different treatment methods leads to differences in number of platelets in PTRP. Up till now, there is no ideal dosage number of PRP, many authors state that the wholeness of platelets is more important than its concentration rate $[6,7]$.

In the study, the concentrations of EGF and VEGF in PRP after activation increased significantly higher than before activation, $\mathrm{p}<0.05$. The GF increasing trend is recorded in many researches but the concentration differs, largely due to different PRP harvest methods, leading to change in number of platelets $[6,7]$.

The above results can affirm that, activated PRP has released not only EGF and VEGF but also a large number of other GFs that affects the wound healing process. Activated platelets leads to the lysis of $\alpha$ particles inside them, from there release antiinflammatory cytokines, chemokines and other important GFs in the wound healing process [12,13]. The increase in concentration of markers in our research serves as base for us to explain the working mechanism of PRP in chronic wounds.

4.2.2. Changement of EGF and VEGF concentration in wound

4.2.2.1. Change of EGF concentration in wound

EGF is the most common GF in wound healing, creating conditions for epithelization(promoted epidermis and ECM layer ) by stimulating the reproduction and movement of keratinocytes; endothelial cells and fibroblasts $[21,22]$ are responsible for increasing the tensil estrength of new skin [23]. Keratinized cells from wound edge of from hair follicles and dermal glands are activated for migration, then reproduction, differentiation, and increase in GF production to increase epithelization in order to restore the epidermis [16]. In chronic wounds, EGF decreases [19], EGF receptors are in incorrect locations (inside cytoplasm 
of keratinocytes instead of membrane) can cause suppression of epithelization [16].

In the research, EGF at tissue tended to increase during treatment, showing the restoration of the epithelizing process, and is clear, objective evidence of the woundhealing stimulation effects of PRP. EGF concentration change at wound location is also accurate on a clinical scale (increased epithelization at wound edge).

\subsubsection{Changement of VEGF concentration in wound tissue}

After the inflammation phase of wound healing is the proliferating phase with strong cellular proliferation forming and recreating extracellular matrix (ECM), increase in vein creation. VEGF is one of the most important proangiogen molecules towards woundhealing. VEGG increases vascular permeability by more than a thousand times compared to histamine [24[; causing increase inregenerate blood vessels and stimulate endothelial cellular functions (proliferation, migration, differentiation, and survival), which are necessary for the forming of new veins $[16 ; 24]$. VEGF is a specific mitogen vascular endothelial cells, monitoring some endothelial integrin receptors during the process of creating new veins [25].

In our study, VEGF concentration in wound tissue has a trend to increase during treatment. This is crucial evidence regarding the effectiveness of PRP towards healing chronic wounds.

Other studies on chronic wounds have shown, skin tissue VEGF start to increase 1 day after occurring injuries; compared to normal skin, VEGF increases dramatically after 3 and 5 days and return to its original condition in around 7-14 days. Low VEGF concentration rates leads to insufficient wound circulation in those with chronic wounds. There is a strong relation between VEGF rate or activity and the concentration of granulation tissue formed in both normal animals and patients with diabetes [24]. In the research, increase of VEGF leads to the increase in the number of veins at wound location. The forming and growth of new veins from existing ones helps to restore blood to damaged tissues; providing oxygen and necessary nutrients to support the growth and restoration of cellular function [17]. The change in VEGF concentration also matches that on a clinical scale. After treatment, the number of wounds with granular tissue have increased, characteristics of good granular increase, covering fully muscle and tendo expose .

\subsubsection{Change in hydroxyproline concentration}

The basic phenomenon of the proliferating phase includes epithelization, increase vessels regeneration, fibroblasts increase synthesis of extracellular matrix (namely collagen). Chronic wounds have an unbalance between the synthesis and decomposition of collagen due to the unbalance between proteinase and chemicals that suppress them, which is caused by aging of fibroblasts [17, 26, 27]. Hydroxyproline is a non-essential amino acid found in collagen, playing a vital role in the synthesis and thermodynamic stabilization of collagen's three-dimensional helix structure. Hydroxyproline which is specific for collagen, is considered a biochemical marker, and reflects the synthesis of collagen in wounds [28, 29].

In the research, at first thehydroxyproline concentration in chronic wounds are low, then it increased dramatically after 2 weeks of treatment by the PRP method $(\mathrm{p}<0.05)$. The increase in hydroxyproline concentration 
reflects the increase of fibroblast number and functions in wounds (base for wounds with the ability to heal), and also reflects the healing of chronic wounds, showing the effectiveness of PRP.

As can be seen, the change in EGF, VEGF and hydroxyproline shows the effectiveness of PRP in wound-healing stimulation.

\section{-Wound-healing mechanism of PRP}

PRP contains many different cytokines and GFs (especially EGF, PDGF, VEGF; GFs play an important role in all phases of wound-healing); attracting progenitor cells to stimulate proliferation and cellular differentiation, enhance the wound healing process through the autocrine and paracrine mechanisms. PDGF, TGF- $\beta$, VEGF and EGF increase three-to-seven-fold in PRP. GFs have kinetic chemical characteristics and stimulate cellular division, monitoring important tissue healing processes, including increasing cellular growth, kinetic chemistry, migration, cellular differentiation, and synthesizing extracellular matrix. GFs in PRP stimulate endothelial cells to grow and form new capillaries $[6,7,12]$.

Tatiana N. (2012) has identified a new peptide in PRP with the capability to increase by $50 \%$ the endothelial cell formation, and increase by $250 \%$ vein-formation functions and by threefold the movements of epithelial cells to meet the needs of the wounds [30].

Activated platelets also releases a great number of other factors such as fibrinogen, serotonin, fibronectin, V, VIII factors and calcium, reducing platelet concentration rate, creating platelets' stability using fibrin and glycoprotein cross-connections. Fibrin when is created promote the intrusion of mono leukocytes, fibroblasts and other progenitor cells play a vital in the wound healing $[6,7]$.

\subsubsection{Changement of MMP12 concentration}

MMP causes degradation of GFs and cytokines (including exogenous EGF, and EGF- receptor) obstructing wound healing in chronic ulcers/wound. A sign of a wound becoming chronic is the prolonged inflammation, due to the increase of preinflammation cytokines and high metalloprotese activities (while TIMP1 decreases), causing the decomposition of collagen. The metalloproteinase concentration rate in wounds signifies the time and chronic severity [19; 31]. Prolonged inflammation creates multiple inflamed cells and cytokines, decreasing the response rate of GFs. Therefore, a solution is to change the microenvironment that is slowing down the healing process, creating a recreation phase and limit the previous inflammation phase [6; 7]. MMP-12 (Macrophage metalloelastase) is not just a pre-inflammation marker of macrophages but is also a pre-inflammation protease; affecting substrates including elastin, fibronectin, Collagen IV. It suppresses the wound-healing and veinforming processes in vitro and in vivo, enhancing the apoptosis process of endothelial cells. Moreover, MMP12 is also involved in the mechanisms of many other cytokines (TGF $\beta, \quad \mathrm{TNF} \alpha, \quad \mathrm{NF} \kappa \mathrm{B}$ ), participating in the inflammation phase and monitoring the formation of veins [32; 33]. In acute corneal epithelial wounds, MMP12 appears early on, with its highest level at 8 hours after injury, then decreasing after 4 and 8 days since injury. MMP12 effects the repairing speed and movement of epithelial cells. The lengthy increase of MMP12 reflects prolonged inflammation, slowing down the healing of wounds [34]. 
In the research, before applying the PRP treatment, the MMP12 concentration was at its highest. The MMP12 concentration shows a decreasing trend after 1-2 weeks of treatment $(\mathrm{p}<0.05)$. After 2 weeks, the concentration is significantly lower (nearly 10 times compared to that after 1 week and 2 weeks, $\mathrm{p}<0.001)$. This is an objective factor showing the decrease in inflammation response. Change in MMP12 concentration matches the inflammation decrease in wounds on a clinical scale.

-Anti-inflammatory mechanism of PRP

The inflammation decrease at wound location is noted after 1 week of treatment. PRP restricts inflammation due to cytokinesuppression and improving the reproduction process by enhancing the formation of new veins and epithelization [35]. This is also related to the GFs in PRP including $\mathrm{HGF}$, PDGF, IGF, TGF - $\beta$, EGF, FGF [35, 36, 37]. Asfaha NC has shown the pain-relieving effectiveness of PRP through PAR4. PRP has a high RANTES and LXA4 concentration rate, which acts as an anti-inflammatory substance [7].

\subsection{Results of PRP treatment}

Study results have shown: $100 \%$ of ulcers are fully healed, average time of treatment is $35,7 \pm 16,9$ days; of which: 4 patients selfhealed, while 20 patients are given skin transplant surgery. All the patients have a medical history, and have a common status such as corrosion, etc. Moreover, patients in the research group have long-existing ulcer, previously treated by traditional methods but are ineffective. The application of the treatment has contributed to healing wound, creating good conditions for the success of surgery.

\section{CONCLUSION}

\section{Chronic wound characteristics}

$100 \%$ of patients had comorbidity among them pressure ulcers had high rate: 21 patients $(70 \%)$, spinal cord injuries had high percentage $(56,7 \%)$; sacrum ulcers had the highest percentage $(66,7 \%)$. Average existing length of ulcers was 8,6 months (range: 1 month to 1 year). Chronic wound site shown chacracteristics as infiltration, callus, Inflammation, congestion and unhealthy granulationtissue. PRP therapy helpedto decrease inflammation as well as stimulate epithelization and tissue recreation. All chronic wounds wereclosed after PRP therapy

2. Changement of Biomarker in activated PRP and in chronic wound tissue after PRP therapy

- The EGF, VEGF concentration in activated PRP increased significantly higher than unactived PRP.

-Autologous PRP therapyhad a beneficial effect on cutaneous regeneration and wound healing of the chronic wound. The autologous PRP promoted the wound healing process by EGF, VEGF increase and MMP12 reduction.

\section{REFERENCES}

1. Mathieu D., Linke J.C., Wattel F. (2006); "Non-healing wounds". In: Handbook on hyperbaric medicine, Mathieu D.E. Netherlands: Springer, 2006, pp. 401-427.

2. Menke N.B., Ward K.R., Witten T.M., Bonchev D.G., Diegelmann R.F. (2007); "Impaired healing". ClinDermatol. 2007, 25:19-25

3. Dowsett C.(2015); "Breaking the cycle of hard-to-heal wounds: balancing cost and care". Wounds International 2015, Vol 6 Issue 2 
4. Marta Otero-Viñas and Vincent Falanga (2016); "Mesenchymal Stem Cells in Chronic Wounds: The Spectrum from Basic to Advanced Therapy". Adv Wound Care (New Rochelle) 2016, Apr 1; 5(4): 149-163.

5. Banfi G.(2012); "Platelet rich plasma". Journal of biological regulators and homeostatic agents 2012;26, 1.

6. Amable P.R., Carias, R.B., Teixeira, M.V., da Cruz Pacheco, I., Correa do Amaral R.J., Granjeiro J.M. and Borojevic R. (2013); "Platelet-rich plasma preparation for regenerative medicine: optimization and quantification of cytokines and growth factors". Stem cell research \& therapy, 2013, 4 (3): 67

7. Elena Conde-Montero, Pablo de la CuevaDobao, José MaríaMartínez González (2017) ; "Platelet-rich plasma for the treatment of chronic wounds: evidence to date". Chronic Wound Care Management and Research. 2017, Vol 2017:4 107-120

8. KristerJärbrink, Gao Ni, Henrik Sönnergren, ArturSchmidtchen, Caroline Pang, Ram Bajpai and Josip Ca (2016); "Prevalence and incidence of chronic wounds and related complications: a protocol for a systematic review". Syst Rev. 2016; 5(1): 152

9. National Pressure Ulcer Advisory Panel (NPUAP)(2016); Pressure Injury Stages, http://www.npuap.org/, 2016.

10.Vladimir N. Obolenskiy, Darya A. Ermolova, Leonid A. Laberko (2014); "Efficacy of platelet - rich plasma for the treatment of chronic wounds". The EWMA Journal, volume 14, No 1, April, 2014, p. 3740.

11.Mehrannia M., Vaezi M., Yousefshahi F., Rouhipour N (2014); "Platelet rich plasma for treatment of nonhealing diabetic foot ulcers:a case report". CJD (canadian journal of diabetes), February 2014, Volume 38, Issue 1, Pages 5 - 8.

12.Javier Ramos-Torrecillas, Elvira De LunaBertos, Olga García-Martínez (2014); "Clinical Utility of Growth Factors and Platelet-Rich Plasma in Tissue Regeneration: A Review". Wounds. 2014; 26(7):207-213
13.Diegelmann R.F. and Evans M.C (2020); "Wound Healing: An Overview of acute, fibronetic and delayed healing". Frontiers in Bioscience 9, 283-289

14.George Han and Roger Ceilley (2017); "Chronic Wound Healing: A Review of Current Management and Treatments". AdvTher. 2017; 34(3): 599-610.

15.Sabine Werner and Richard (2003); Regulation of Wound Healing by Growth Factors and Cytokines; GrosPhysiol Rev 83: 835-870, 2003;

16.Stephan Barrientos; OliveraStojadinovic, Michael S. Golinko, Harold Brem, MarjanaTomic-Canic (2008), Growth factors and cytokines in wound healing; Wound Repair and Regeneration Wound, (2008), 16, 585-601

17.Hart J. (2002); Inflammation. 1: Its role in the healing of acute wounds. $J$ Wound Care. 2002 Jun; 11(6):205-9.

18.Zhang Y, Wang T, Dong J (2016); Growth factor therapy in patients with partialthickness burns: a systematic review and meta-analysis. Wound J. 2016 Jun; 13(3): 354-66.

19.ShoYamakawa \& Kenji Hayashida (2019), Advances in surgical applications of growth factors for wound healing; Burns \& Trauma, volume 7 , Article number: 10 (2019).

20.Park JW, Hwang SR, Yoon IS (2017); Advanced growth factor delivery systems in wound management and skin regeneration. Molecules. 2017; 22:E1259.

21.Nanney LB (1990); Epidermal and dermal effects of epidermal growth factor during wound repair. J Invest Dermatol. 1990; 94:624-9.

22.J.Hardwicke, D.Schmaljohann, D.Boyce, D.Thomas (2008); Epidermal growth factor therapy and wound healing - past, present and future perspectives; The Surgeon Volume 6, Issue 3, June 2008, Pages 172-177

23. Brown GL, Curtsinger LJ, White M, Mitchell RO, Pietsch J, Nordquist R, et al. (1988); Acceleration of tensile strength of incisions treated with EGF and TGF-beta. Ann 
Surg. 1988; 208:788-94.

24.Kelly E. Johnson and Traci A. Wilgus (2014); Vascular Endothelial Growth Factor and Angiogenesis in the Regulation of Cutaneous Wound Repair; Adv Wound Care. 2014 Oct 1; 3(10): 647-661.

25.Thittamaranahalli

Muguregowda

Honnegowda, Pramod Kumar, Echalasara Govindarama Padmanabha Udupa,Sudesh Kumar, Udaya Kumar, Pragna Rao (2015); Role of angiogenesis and angiogenic factors in acute and chronic wound healing; Plast Aesthet Res 2015; 2:243-249

26.George Han and Roger Ceilley (2017); Chronic Wound Healing: A Review of Current Management and Treatments; $A d v$ Ther. 2017; 34(3): 599-610.

27.Robert Nunan, Harding K. G. and Martin P. (2014); "Clinical challenges of chronic wounds: searching for an optimal animal model to recapitulate their complexity"; Disease Models \& Mechanisms 7, 1205-1213

28.Amit Kuma Srivastava, PiushKhare, NavdeepRaghuwanshi et al (2016); Hydroxyproline: A Potential Biochemical Marker and Its Role in the Pathogenesis of Different Diseases. Curr Protein Pept Sci. 2016; 17(6):596-602

29.Guilherme F. Caetano, MarcioFronza, Marcel N. Leite, Ary Gomes \&Marco Andrey Cipriani Frade (2016); Comparison of collagen content in skin wounds evaluated by biochemical assay and by computer-aided histomorphometric analysis; Pharm Biol. 2016 Nov; 54(11):2555-2559

30.Tatiana N. Demidova-Rice, Lindsey Wolf, Jeffry Deckenback, Michael R. Hamblin, Ira M. Herman (2012). "Human PlateletRich Plasma- and Extracellular MatrixDerived Peptides Promote Impaired Cutaneous Wound Healing In vivo”. PLOS. https://doi.org/10.1371/journal.pone.0032146
31.Krister Järbrink, Gao Ni, Henrik Sönnergren, Artur Schmidtchen, Caroline Pang, Ram Bajpai and Josip Ca (2016);"Prevalence and incidence of chronic wounds and related complications: a protocol for a systematic review". Syst Rev. 2016; 5(1): 152

32.Verma RP, Hansch C (March 2007); "Matrix metalloproteinases (MMPs): chemical-biological functions and (Q)SARs". Bio org. Med. Chem. 15 (6): 222368.

33.Mikel Aristorena, Eunate Gallardo-Vara, MatejVicen et al. (2019); MMP-12, Secreted by Pro-Inflammatory Macrophages, Targets Endoglin in Human Macrophages and Endothelial Cells; Int. J. Mol. Sci. 2019, 20, 3107;

34.Marie Wolf, Inna Maltseva, Selene M. Clay, Peipei Pan, Abhinay Gajjala and Matilda F. Chan (2017); Effects of MMP12 on cell motility and inflammation during corneal epithelial repair; Exp Eye Res. 2017 Jul; 160: 11-20.

35.Manish

Suthar, Saniya

Gupta, SuhailBukhari and Venkatesh

Ponemone (2017); Treatment of chronic nonhealing ulcers using autologous platelet rich plasma: a case series. J Biomed Sci. 2017; 24 : 16

36.Hamilton B., Tol J.L. Knez W. and Chalabi H. (2013); "Exercise and the platelet activator calcium chloride both influence the growth factor content of platelet-rich plasma (PRP): overlooked biochemical factors that could influence PRP treatment". British journal of sports medicine, $2015 \mathrm{Jul} ; 49(14): 957-60$

37.Marx R.E (2004). "Platelet-rich plasma: evidence to support its use". J Oral axillo fac Surg. 2004. 62:489-496 